\title{
Comparative study of bacampicillin and ampicillin in the treatment of uncomplicated gonorrhoea*
}

\author{
PATRICIA J SPENGLER AND LARRY D EDWARDS \\ From the Biomedical Sciences Department, University of Illinois, Rockford, Illinois, USA
}

SUMMARY Bacampicillin hydrochloride, a pro-drug ester of ampicillin trihydrate which is hydrolysed to ampicillin after absorption, was used in a randomised comparative study of ampicillin $3.5 \mathrm{~g}$ and bacampicillin $1.6 \mathrm{~g}$ (each with probenecid $1 \mathrm{~g}$ ) in the treatment of uncomplicated gonorrhoea. This dose of bacampicillin was selected because in serum it gives approximately the same peak concentration of ampicillin as $3.5 \mathrm{~g}$ of the parent drug. Genital, pharyngeal, and anal cultures were performed at the initial visit and at follow up 5-9 days after treatment. There was no statistically significant difference in the microbiological or clinical response of patients with genital gonorrhoea who were treated with ampicillin and those treated with bacampicillin. At 5-9 days after treatment, $93 \%$ of the ampicillin-treated patients and $89 \%$ of the bacampicillin-treated patients had negative genital, pharyngeal, and anal culture results; furthermore, $87 \%$ and $89 \%$ respectively had no symptoms of infection. Fewer gastrointestinal side effects were noted in the patients treated with bacampicillin.

\section{Introduction}

Oral antibiotic therapy has the advantages of greater patient co-operation and a lower rate of hypersensitivity reactions $;{ }^{12}$ moreover, there is a need for effective oral antibiotics which have the potential for eradicating gonorrhoea from the pharynx as well as from anogenital sites.

Several investigators have reported high pharyngeal colonisation rates, particularly in women and homosexual men. In studies by Wiesner et $a l^{3}$ and Bro-Jorgensen and Jensen ${ }^{4}$ of patients who had positive culture results for Neisseria gonorrhoeae at genital, rectal, or pharyngeal sites, pharyngeal colonisation rates ranged from $10-10 \cdot 3 \%$ in women, $3 \cdot 2-6 \%$ in male heterosexuals, and $20 \cdot 9-25 \%$ in male homosexuals. ${ }^{34}$ In a population known to have infrequent orogenital sexual contact, Noble et $a l^{5}$ found that among patients with concurrent genital or rectal gonorrhoea $3 \cdot 3 \%$ of the men and $1.9 \%$ of the women had gonococcal colonisation of the pharynx. In a recent study by Gartner and Edwards (paper presented at 79th Annual Meeting, American Society for Microbiology, Los Angeles-Honolulu, 1979)

\footnotetext{
*This study was presented in part at the 79th Annual Meeting of the American Society for Microbiology, Los Angeles-Honolulu, May 1979
}

Address for reprints: Dr L D Edwards, Biomedical Sciences Department, Division of Infectious Diseases, Rockford School of Medicine, 1601 Parkview, Rockford, Illinois 61101, USA

Received for publication 21 August 1979 multiple cultures were obtained from each potential site of infection-pharynx, anus, and genital tract-before treatment. Patients were selected for the study if they had either a positive result to a genital smear or a previous positive genital culture result. In this study $23 \cdot 8 \%(10 / 42)$ of heterosexual women and $8.9 \%(4 / 45)$ of heterosexual men who were culture-positive at a genital or an anal site also had a positive result to a pharyngeal culture.

Bro-Jørgensen and Jensen ${ }^{4}$ and Karney et $a l^{6}$ demonstrated that $N$ gonorrhoeae is difficult to eradicate from the pharynx with the multiple antibiotic regimens that they tried. The Centre for Disease Control in Atlanta, Georgia, does not recommend the use of the standard oral regimen for treating genital gonorrhoea (ampicillin $3.5 \mathrm{~g}$ with probenecid $1 \mathrm{~g}$ ) for the treatment of pharyngeal gonorrhoea. ${ }^{7}$ However, in a recent study by $\mathrm{Di}$ Caprio $^{8}$ success rates of $96 \cdot 1-98 \cdot 7 \%$ in the treatment of pharyngeal gonorrhoea were achieved with ampicillin trihydrate $3.5 \mathrm{~g}$ with probenecid $1 \mathrm{~g}$ initially, followed by $500 \mathrm{mg}$ four times daily for two days, a total of $7 \cdot 5 \mathrm{~g}$ of ampicillin.

In view of the need for oral antibiotics which can eradicate pharyngeal as well as genital gonorrhoea, we decided to study bacampicillin hydrochloride, an oral preparation which is a pro-drug ester of ampicillin trihydrate. Bacampicillin, after absorption, is hydrolysed to ampicillin and gives serum concentrations two to three times higher than an equimolar dose of ampicillin trihydrate. ${ }^{910}$ 


\section{Patients and methods}

\section{STUDY POPULATION}

Sixty-one men and 22 women with culture-positive uncomplicated gonorrhoea were randomly assigned to one of two oral treatment groups. One group was treated with ampicillin $3.5 \mathrm{~g}$, the other with bacampicillin $1.6 \mathrm{~g}$, a one-third equimolar dose. Probenecid $1 \mathrm{~g}$ was administered simultaneously with each drug.

The patients were volunteers aged 18 years or more, who attended the sexually transmitted diseases clinic at the. Winnebago County Department of Public Health, Rockford, Illinois, USA. Those patients who were pregnant or nursing an infant, wished to donate blood during the study period, were known or suspected to be allergic to penicillin or cephalosporins, had hepatic disease or impaired renal function, required other concomitant antimicrobial therapy, had received systemic antimicrobial therapy within the previous month, or had infectious mononucleosis, impaired immunological function, syphilis, or chronic urinary tract infection were excluded from the study.

\section{COLLECTION OF SPECIMENS}

At the initial visit, urethral and endocervical specimens were obtained by calcium alginate swabs from men and women respectively for Gram stain and culture. Pharyngeal and anal specimens were obtained for culture from all patients. To monitor adverse drug reactions, total blood counts, urine analyses, and serum analyses for hepatic and renal function (bilirubin, alkaline phosphatase, creatinine, glutamic oxalacetic transaminase, glutamic pyruvic transaminase, and urea nitrogen) were performed. Routine serological Venereal Disease Research Laboratory (VDRL) tests for syphilis were performed; patients with reactive VDRL test results were excluded from the study. Gram stains, freshly centrifuged urine sediment specimens, and salinediluted wet-mount preparations of vaginal secretions were examined microscopically for yeast forms and trichomonads. Patients with apparent candidosis or trichomoniasis were not included in the study. Two hours after treatment, specimens of urine, blood, and saliva were collected for determination of ampicillin concentrations.

Follow-up visits took place 5-9 days after treatment, at which time patients were questioned about symptoms and possible drug-related reactions. Gram stains, cultures, wet-mount preparations, urine analyses, and tests to monitor for adverse drug reactions were repeated as for the initial visit. The procedures followed were in accordance with the ethical standards of the committees on human experimentation of the University of Illinois.

\section{DIAGNOSIS}

All cultures were inoculated directly on to modified Thayer-Martin medium (Baltimore Biological Laboratories, Cockeysville, Maryland) containing vancomycin, colistin, nystatin, and trimethoprim ${ }^{11}$ and were incubated immediately at $35^{\circ} \mathrm{C}$ in $5-10 \%$ carbon dioxide. Cultures were transported to the Rockford School of Medicine laboratories where standard procedures for isolation and identification of $N$ gonorrhoeae were followed with confirmation of pharyngeal and anal isolates by sugar fermentation tests. ${ }^{12}$

\section{ANTIBIOTIC SENSITIVITIES}

Minimum inhibitory concentrations (MICs) of ampicillin for $N$ gonorrhoeae isolates were determined by the agar dilution technique. ${ }^{13} \mathrm{~A}$ suspension of $10^{5}$ organisms per $\mathrm{ml}$ in MuellerHinton broth supplemented by $1 \%$ IsoVitaleX (Baltimore Biological Laboratories, Cockeysville, Maryland) was delivered by automatic inoculator to the surface of each agar plate with antibiotic concentrations from 0.049 to $100.0 \mu \mathrm{g} / \mathrm{ml}$. The ampicillin-containing agar plates were prepared just before use in GC medium base (Difco Laboratories, Detroit, Michigan) with $1 \%$ IsoVitaleX. The MIC was defined as the dilution with no visible growth after 48 hours of incubation.

MICs of penicillin were not determined.

\section{B-LACTAMASE PRODUCTION}

Fresh subcultures of $N$ gonorrhoeae on chocolate agar were tested for $\beta$-lactamase production using the chromogenic cephalosporin method. ${ }^{14}$

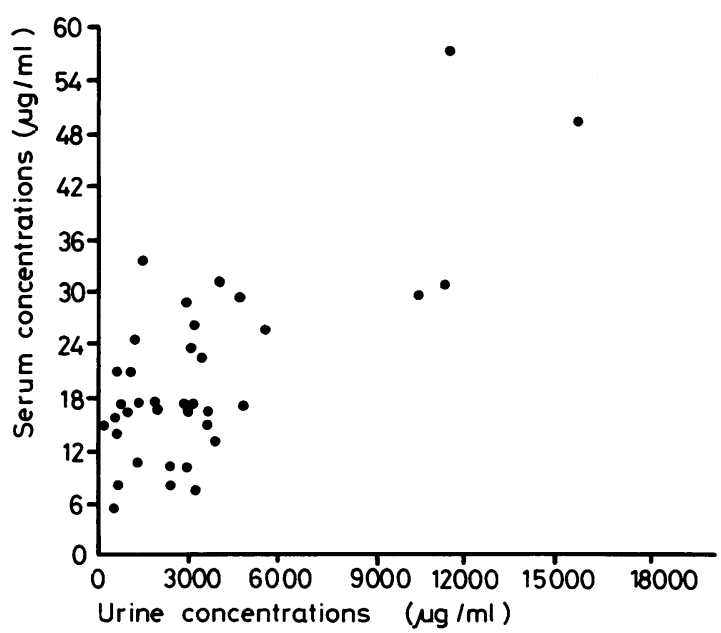

FIG 1 Correlation of ampicillin concentrations in serum and urine specimens from 37 patients two hours after administration of ampicillin $3.5 \mathrm{~g}$ with probenecid $1 \mathrm{~g}$. 
ANTIMICROBIAL ASSAY FOR AMPICILLIN CONCENTRATIONS

Specimens of urine, blood, and saliva were frozen immediately and transported to the Rockford School of Medicine laboratories, where they were stored at $-20^{\circ} \mathrm{C}$. Specimens were tested for drug concentrations within 72 hours of collection by agar-well bioassay $^{15}$ using Bacillus subtilis as the indicator organism.

\section{STATISTICAL METHODS}

$\chi^{2}$ analysis with Yates's correction was used for statistical comparisons of proportions and Student's $t$ test for unpaired data (one-tailed) for comparisons of means. Correlation analyses (figs 1 and 2) were computed by Pearson product-moment correlation coefficients using the one-tailed Student's $t$ test for unpaired data to determine levels of significance.

\section{Results}

\section{PATIENT POPULATION}

Of the 83 patients treated, $57(69 \%)$ returned for a follow-up visit. The treatment groups were statistically similar with regard to sex, race, history of previous infection, and age (table I).

The study population differed from our regular clinic population in that it was predominantly male whereas the latter was equally divided between men and women. More men than women volunteered for the study, and women who were possibly pregnant were excluded. Racial distribution was representative of the regular clinic population; both the study group and the regular clinic population were approximately $50 \%$ white and $50 \%$ non-white.

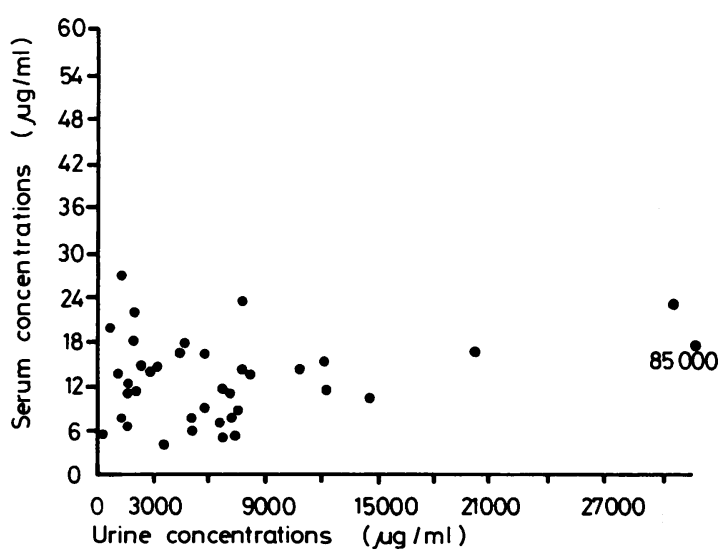

FIG 2 Correlation of ampicillin concentrations in serum and urine specimens from 38 patients two hours after administration of bacampicillin $1.6 \mathrm{~g}$ with probenecid $1 \mathrm{~g}$.
TABLE I Characteristics of patients treated for gonorrhoea with ampicillin and bacampicillin

\begin{tabular}{|c|c|c|}
\hline \multirow[b]{2}{*}{ Characteristics } & \multicolumn{2}{|c|}{ Treatment group } \\
\hline & $\begin{array}{l}\text { Ampicillin } \\
(n=30)\end{array}$ & $\begin{array}{l}\text { Bacampicillin } \\
(n=27)\end{array}$ \\
\hline \multicolumn{3}{|l|}{ Sex } \\
\hline Male & 21 & 22 \\
\hline Female & 9 & 5 \\
\hline \multicolumn{3}{|l|}{ Race } \\
\hline White & 14 & 11 \\
\hline Non-white & 16 & 16 \\
\hline \multicolumn{3}{|l|}{ Previous gonococcal } \\
\hline $\begin{array}{l}\text { infection } \\
\text { Mean age (years) }\end{array}$ & 14 & 15 \\
\hline Mean age (years) & $24 \cdot 1$ & $23 \cdot 9$ \\
\hline
\end{tabular}

Two men in the study group stated that they were homosexuals and one stated that he was bisexual; the rest of the patients claimed to be practising heterosexuals.

\section{GRAM STAINS AND CULTURES}

At the time of the initial visit, intracellular Gramnegative diplococci typical of $N$ gonorrhoeae were identified on Gram stains of urethral exudate of all 43 men and on Gram stains of endocervical exudate of $11(79 \%)$ of the 14 women. Three women who entered the study as named contacts of men with confirmed gonococcal urethritis had negative Gram stains and positive endocervical culture results.

Initially, all patients had positive genital culture results and negative pharyngeal culture results. Only one patient, female, had a positive result to an anal culture.

MICs

MICs for all 58 isolates taken at the initial visit are shown in table II. There was no significant difference in the distribution of MICs between the group treated with ampicillin and that treated with bacampicillin $\left(\chi_{4}{ }^{2}=3 \cdot 63, \mathrm{P} \cong 0 \cdot 46\right)$.

TABLE II Minimum inhibitory concentrations of ampicillin for 58 isolates of Neisseria gonorrhoeae obtained at patients' initial visits

\begin{tabular}{|c|c|c|c|c|c|c|}
\hline \multirow[b]{3}{*}{$\begin{array}{l}M I C s \\
(\mu \mathrm{g} / \mathrm{ml}\end{array}$} & \multicolumn{6}{|c|}{ Isolates from patient-groups } \\
\hline & \multicolumn{2}{|c|}{ Ampicillin $(n=31)$} & \multicolumn{2}{|c|}{ Bacampicillin $(n=27)$} & \multicolumn{2}{|l|}{ Total } \\
\hline & No & $\%$ & No & $\%$ & No & $\%$ \\
\hline $\begin{array}{l}0.049 \\
0.098 \\
0.195 \\
0.390 \\
0.780\end{array}$ & $\begin{array}{r}10 \\
8 \\
3 \\
9 \\
1\end{array}$ & $\begin{array}{r}32 \cdot 2 \\
25 \cdot 8 \\
9 \cdot 7 \\
29 \cdot 0 \\
3 \cdot 2\end{array}$ & $\begin{array}{r}12 \\
6 \\
5 \\
4 \\
0\end{array}$ & $\begin{array}{r}44 \cdot 4 \\
22 \cdot 2 \\
18 \cdot 5 \\
14 \cdot 8 \\
0\end{array}$ & $\begin{array}{r}22 \\
14 \\
8 \\
13 \\
1\end{array}$ & $\begin{array}{r}37 \cdot 9 \\
24 \cdot 1 \\
13 \cdot 8 \\
22 \cdot 4 \\
1 \cdot 7\end{array}$ \\
\hline $\begin{array}{l}\text { Mean } \\
\text { Median }\end{array}$ & $\begin{array}{l}0 \cdot 104 \\
0 \cdot 198\end{array}$ & & $\begin{array}{l}0.086 \\
0.137\end{array}$ & & $\begin{array}{l}0.170 \\
0.098\end{array}$ & \\
\hline
\end{tabular}


$\beta$-LACTAMASE PRODUCTION

No isolates produced $\beta$-lactamase.

\section{DRUG CONCENTRATIONS}

For 37 patients treated with ampicillin (fig 1), the serum ampicillin concentrations at two hours ranged from $5 \cdot 8-58.0 \mu \mathrm{g} / \mathrm{ml}$ with a median value of $18 \cdot 3 \mu \mathrm{g} / \mathrm{ml}$ and a mean value of $21 \cdot 1 \mu \mathrm{g} / \mathrm{ml}$; urine concentrations ranged from $370 \cdot 0-15400 \mu \mathrm{g} / \mathrm{ml}$ with median and mean values of 2595 and $3364 \cdot 6 \mu \mathrm{g} / \mathrm{ml}$ respectively.

For 38 patients treated with bacampicillin (fig 2), the serum ampicillin concentrations at two hours ranged from $4 \cdot 7-28 \mu \mathrm{g} / \mathrm{ml}$ with median and mean values of $13.6 \mu \mathrm{g} / \mathrm{ml}$; urine concentrations ranged from 700-85 $000 \mu \mathrm{g} / \mathrm{ml}$ with median and mean values of $5220 \mu \mathrm{g} / \mathrm{ml}$ and $8292 \cdot 5 \mu \mathrm{g} / \mathrm{ml}$ respectively.

No ampicillin was detected in saliva specimens from patients in either treatment group.

There was a strong positive correlation between serum and urine concentrations in the ampicillintreated patients $(r=0 \cdot 72, \mathrm{P}<0 \cdot 001)$. Concentrations of ampicillin in the serum and urine of bacampicillintreated patients showed no significant correlation $(r=0 \cdot 18, \mathrm{P} \cong 0 \cdot 14)$.

Serum drug concentrations at two hours in the ampicillin-treated patients were significantly higher than those in the bacampicillin-treated group $(t=3 \cdot 70, \mathrm{P}<0 \cdot 001)$ and urine concentrations were significantly lower $(t=-2 \cdot 10, \mathrm{P}<0 \cdot 025)$.

\section{MICROBIOLOGICAL RESPONSE}

The microbiological response was similar in the two groups $\left(\chi_{1}^{2}=0.02, \mathrm{P} \bumpeq 0.9\right)$. Ninety-three per cent $(28 / 31)$ of the ampicillin-treated patients and $89 \%$ $(24 / 27)$ of the bacampicillin-treated patients had negative culture results at follow up, including the female patient whose initial anal culture gave a positive result. Five heterosexual patients, four male and one female, who were initially culture-positive at genital sites, were culture-positive 5-9 days after treatment. Four had positive results at genital sites; one male patient had a positive pharyngeal culture result after treatment although his initial pharyngeal culture had a negative result. On examination and interview during the follow-up visit, all five of these patients were asymptomatic.

For the five patients who were culture-positive after treatment, no significant difference was noted between MICs on initial and post-treatment isolates (table III).

The four men, including the one with a positive pharyngeal culture result, admitted to further contact during the study period and may represent reinfections rather than treatment failures.

Gram stains gave positive results for the three men
TABLE III MICs of ampicillin for $N$ gonorrhoeae isolates from five patients at initial visit and at follow up (before and after treatment)

\begin{tabular}{lllll}
\hline & & & \multicolumn{2}{l}{$M I C(\mu \mathrm{g} / \mathrm{ml})$} \\
\cline { 4 - 5 } Patient No & Sex & Drug & $\begin{array}{l}\text { Initial } \\
\text { visit }\end{array}$ & $\begin{array}{l}\text { Follow-up } \\
\text { visit }\end{array}$ \\
\hline 1 & M & Bacampicillin & $0 \cdot 390$ & $0 \cdot 780$ \\
2 & M & Ampicillin & $0 \cdot 195$ & $0 \cdot 390$ \\
3 & M & Bäampicillin & $0 \cdot 049$ & $0 \cdot 049$ \\
4 & F & Ampicillin & $0 \cdot 098$ & $0 \cdot 390$ \\
5 & M & Bacampicillin & $0 \cdot 390$ & $0 \cdot 390^{*}$ \\
\hline
\end{tabular}

*Pharyngeal isolate

with positive urethral culture results after treatment. These three patients, two initially treated with bacampicillin and one treated with ampicillin, and the man with a positive pharyngeal culture result, who was initially treated with bacampicillin, responded satisfactorily to retreatment with intramuscular spectinomycin or aqueous procaine penicillin $\mathrm{G}$. The culture-positive female patient had a negative Gramstain result and therefore was not immediately retreated. After culture results were known, we were unable to locate this patient.

\section{CLINICAL RESPONSE}

There was no statistical difference between the treatment groups in clinical response at 5-9 days $\left(\chi_{1}^{2}=0.02, P \stackrel{\wedge}{=} 0.9\right)$. Eighty-seven per cent $(26 / 30)$ of the patients treated with ampicillin and $89 \%(24 / 27)$ of those treated with bacampicillin had no symptoms at follow up. Seven patients $(13 \cdot 3 \%$ of those treated with ampicillin and $11 \cdot 1 \%$ of those treated with bacampicillin) had symptoms after treatment, all of whom were culture-negative. Of these, four were women who complained of vaginal discharge; one had candidosis, one trichomoniasis, and two unexplained vaginal discharge. Three men had either dysuria, discharge, or frequency of micturition.

\section{ADVERSE REACTIONS}

Adverse reactions occurred more commonly in the ampicillin group $(23 \cdot 3 \% ; 7 / 30)$ than in the bacampicillin group $(3 \cdot 7 \% ; 1 / 27)\left(\chi_{1}^{2}=3 \cdot 06, \mathrm{P} \cong 0.08\right)$. Five patients had diarrhoea with four to six stools per day for one or two days; two had mild nausea or epigastric discomfort. One patient treated with ampicillin experienced globus hystericus. Haematological tests, urine analyses, and tests for renal and hepatic function showed no drug-related abnormalities.

\section{Discussion}

Studies have shown that a one-third equimolar dose of bacampicillin ( $1.6 \mathrm{~g})$ results in approximately the 
same peak serum concentrations as ampicillin $3 \cdot 5 \mathrm{~g}$ and that peak ampicillin concentrations are obtained approximately 30 minutes earlier with bacampicillin than with ampicillin. ${ }^{9}{ }^{10}$. Unpublished information supplied by the pharmaceutical company (Pfizer Inc., New York, NY) indicated that peak serum concentrations of ampicillin would be obtained at 1.5 hours with bacampicillin and 2.2 hours with ampicillin when these drugs are administered without probenecid. We were limited to collecting single specimens for drug assays in this outpatient clinic and all our patients received probenecid; thus we chose to collect specimens at two hours. The significantly lower serum concentrations and higher urine concentrations obtained in the bacampicillintreated group suggest that peak serum concentrations occurred earlier in the bacampicillin group than in the ampicillin group.

There was no statistical difference in microbiological response or clinical response between the two treatment groups. Bacampicillin in the dosage used was as effective as the standard regimen of treatment with ampicillin.

The persistence of symptoms in 13\% (7/52) of patients who were culture-negative for $N$ gonorrhoeae after treatment suggests that they were initially infected with multiple micro-organisms. Although we did not perform cultures for Chlamydia trachomatis, Bowie et al ${ }^{16}$ and Holmes et a $l^{17}$ isolated $C$ trachomatis from $18 \%$ and $19 \%$ respectively of men with urethral gonorrhoea, all of whom developed postgonococcal urethritis.

Other micro-organisms have also been implicated as causative agents of nongonococcal urethritis, cervicitis, and vaginitis. In two of the four women with persistent symptoms, candidosis and trichomoniasis were diagnosed at 5-9 days although the presence of yeasts or trichomonads was not noted before treatment.

With the doses used in this study, we were unable to detect ampicillin in the saliva of patients from either treatment group. We could not evaluate the efficacy of bacampicillin in the treatment of pharyngeal gonorrhoea since our study population mainly comprised male heterosexuals, none of whom had pharyngeal colonisation before treatment. The administration of bacampicillin $1.6 \mathrm{~g}$ with probenecid $1 \mathrm{~g}$, however, was tolerated with minimal gastrointestinal side effects and was as effective as the standard oral regimen of ampicillin trihydrate for anogenital gonococcal infection.

Since Di Caprio et al found ampicillin to be effective in treating pharyngeal gonorrhoea when using a dose of $3.5 \mathrm{~g}$ with probenecid $1 \mathrm{~g}$ followed by $500 \mathrm{mg}$ four times daily for two days and we found bacampicillin $1.6 \mathrm{~g}$ to be as effective as the single dose ampicillin-probenecid regimen in genital gonorrhoea, we believe that bacampicillin should be studied at higher doses in a population with a high frequency of orogenital sexual practices to see if adequate salivary concentrations can be obtained and correlated with eradication of $N$ gonorrhoeae in the pharynx.

Grant support was given by Pfizer Pharmaceuticals, New York, USA.

\section{References}

1. Finland $\mathbf{M}$, Weinstein $L$. Complications induced by antimicrobial agents. $N$ Engl $J$ Med 1953;248:220-6.

2. Weinstein L. The complications of antibiotic therapy. Bull NY Acad Med 1955;31:500-18.

3. Wiesner PJ, Tronca E, Bonin P, Pedersen AHB, Holmes KK. Clinical spectrum of pharyngeal gonococcal infection. NEngl J Med 1973;288: 181-5.

4. Bro-Jørgensen A, Jensen T. Gonococcal pharyngeal infections: report of 110 cases. Br J Vener Dis 1973;49:491-9.

5. Noble RC, Cooper RM, Miller BR. Pharyngeal colonisation by Neisseria gonorrhoeae and Neisseria meningitidis in black and white patients attending a venereal disease clinic. $\mathrm{Br} J$ Vener Dis 1979; 55: 14-9.

6. Karney WW, Turck M, Holmes KK. Comparative therapeutic and pharmacological evaluation of amoxycillin and ampicillin plus probenecid for the treatment of gonorrhea. Antimicrob Agents Chemother 1974;5:114-8.

7. Wiesner PJ. Gonococcal pharyngeal infection. Clin Obstet Gynecol 1975; 18: 121-9.

8. Di Caprio JM, Reynolds J, Frank G, Carbone J, Nishimura $\mathrm{R}$ Ampicillin therapy for pharyngeal gonorrhea. JAMA 1978; 239: 1631-3.

9. Magni L, Sjöberg B, Sjövall J, Wessman J. Clinical pharmacological studies with bacampicillin. In: Williams JD, Geddes AM, eds. Chemotherapy: Penicillins and Cephalosporins, Vol 5. New York: Plenum Press, 1976; 109-14.

10. Sjövall J, Magni L, Bergan T. Pharmacokinetics of bacampicillin compared with those of ampicillin, pivampicillin, and amoxycillin. Antimicrob Agents Chemother 1978; 13:90-6.

11. Martin JE, Armstrong JH, Smith PB. New system for cultivation of Neisseria gonorrhoeae. Appl Microbiol 1974; 27:802-5.

12. Kellogg DS. Neisseria gonorrhoeae (gonococcus). In: Lennette E, Spaulding E, Truant J, eds. Manual of Clinical Microbiology. Washington DC: American Society for Microbiology, 1974:124-9.

13. Thornsberry C, Gavan TL, Gerlach EH. New developments in antimicrobial agent susceptibility testing. In: Sherris JC, ed. Cumitech 6. Washington DC: American Society for Microbiology, 1977: 1-13.

14. O'Callaghan C, Morris A, Kirby S, Shingler A. Novel method for detection of $\beta$-lactamases by using a chromogenic cephalosporin substrate. Antimicrob Agents Chemother 1972;1:283-8

15. Winters RE, Litwack KD, Hewitt WL. Relation between dose and levels of gentamicin in blood. J Infect Dis 1971;124 (suppl): $90-5$.

16. Bowie WR, Alexander ER, Holmes KK. Etiologies of postgonococcal urethritis in homosexual and heterosexual men. Sex Transm Dis 1978; 5: 151-4.

17. Holmes KK, Handsfield HH, Wang SP, et al. Etiology of nongonococcal urethritis. N Engl J Med 1975; 292: 1199-205. 